\title{
Neuropeptide $Y$ innervation in the vaginal mucosa among patients with pelvic organ prolapse
}

\author{
JIE-MEI HU ${ }^{1}$, LIN WANG $^{3}$, XIAO CHENG $^{2}$, LI-HUA ZHOU $^{2}$ and ZHI-GANG LI ${ }^{1}$ \\ ${ }^{1}$ Department of Obstetrics and Gynecology, Guangdong General Hospital and Guangdong Academy of Medical Sciences; \\ ${ }^{2}$ Department of Anatomy, Zhong Shan School of Medicine, Sun Yat-Sen University, Guangzhou, Guangdong 510080; \\ ${ }^{3}$ Wuhu Hospital Of Traditional Chinese Medicine, Wuhu, Anhui 241000, P.R. China
}

Received July 14, 2011; Accepted November 9, 2011

DOI: $10.3892 / \mathrm{mmr} .2011 .689$

\begin{abstract}
The purpose of this study was to explore the innervation of neuropeptide Y (NPY) in the anterior vaginal mucosa of menopausal patients suffering from pelvic organ prolapse (POP). To do this, we analyzed the distribution and expression of NPY and its correlation with the occurrence and development of POP. Changes in NPY abundance in the anterior vaginal mucosa were assessed by immunohistochemistry in tissue samples collected from 41 POP patients and 9 control subjects. We divided patients into 4 populations, designated POP IV-POP I, exhibiting decreasing levels of NPY innervation. Through multivariate regression analysis, the level of reduction in NPY innervation was shown to be associated with an increasing severity of POP disease in a statistically significant manner. In conclusion, our data reveal that as the symptoms of POP intensify, the expression of NPY in anterior vaginal mucosa decreases progressively. Menopause, in combination with stress urinary incontinence (SUI), results in a stronger effect on both nerve damage to the pelvic floor and NPY alteration, compared to other risk factors, such as parity and weight.
\end{abstract}

\section{Introduction}

The muscles, fasciae, ligaments and corresponding nerves of the pelvic floor constitute a complex support system that maintains the proper localization of pelvic organs such as the uterus, bladder and rectum. Pelvic organ prolapse (POP), including uterine prolapse, cystocele and rectocele, results from structural defects in, damage to and dysfunction of the pelvic floor support. POP and stress urinary incontinence (SUI) are disorders contributing to female pelvic floor

Correspondence to: Professor Zhi-Gang Li, Department of Obstetrics and Gynecology, Guangdong General Hospital and Guangdong Academy of Medical Sciences, No. 106 Zhongshan Road 2, Guangzhou 510080, P.R. China

E-mail: lizhigang200805@163.com

Key words: pelvic organ prolapse, anterior vaginal wall, neuropeptide $\mathrm{Y}$ dysfunction. POP occurs in 40-50\% of women over 60 years of age and is related to a variety of factors such as vaginal delivery, high body mass index (1) and menopause. Although much has been learned about the treatment of POP through clinical practice, basic research into its pathogenesis remains inadequate. Patients suffering from pelvic floor dysfunction partially lose innervation. Normal pelvic floor tissues have abundant nerve fibers and neuropeptide Y (NPY) (2), which is particularly rich in peptidergic nerve fibers. In this study, we assess the connection between NPY distribution and expression and POP pathogenesis.

\section{Materials and methods}

Research subjects. In this study, we examined 50 clinical cases from patients admitted to our hospital between August 2008 and June 2009. An overview of the research subjects is shown in Table I. Our cases contained 41 POP patients and 9 in the control group (presenting with benign, non-POP and non-SUI gynecological diseases). Based on the POP-quantification (POP-Q) system that was proposed by Bump et al (1995), clinical history and a gynecological examination were used to select those who met the diagnostic criteria of POP research subjects. Patients were classified into 4 stages: the POP I group ( 8 cases), the POP II group (10 cases), the POP III group (15 cases), and the POP IV group ( 8 cases). Evaluation of the patient clinical histories revealed that certain POP patients did not exhibit SUI during early treatments, but they developed SUI symptoms between 4 months and 3 years following POP occurrence, as confirmed by a urodynamic test. These patients were designated POP/SUI. Our control group consisted of patients with benign vaginal tumors who underwent surgery but displayed no POP or SUI. All research subjects met the following criteria: no injuries to the lumbosacral spine or brain, no estrogen drugs taken during the last 3 months, no ovarian tumors, no prior urethral and vaginal surgeries, and no endometriosis based on post-surgery pathology (Table I).

\section{Samples and methods}

Specimen preparation. During vaginal surgery, tissue (3-cm thick, $250 \mathrm{mg}$ ) was cut from an area of the anterior vaginal wall below the urethra. The tissue was washed with saline to remove surface blood, fixed with $10 \%$ neutral formalin, 
Table I. Overview of the research subjects.

\begin{tabular}{lccccr}
\hline Group & Cases & $\begin{array}{c}\text { Age } \\
\text { (years) }\end{array}$ & Menopause & Concurrent SUI & Parity \\
\hline Control & 9 & $58.69 \pm 5.88$ & $2(22 \%)$ & $0(0 \%)$ & $1.22 \pm 0.44$ \\
I & 8 & $60.50 \pm 9.05$ & $6(75 \%)$ & $0(0 \%)$ & $20.74 \pm 2.31$ \\
II & 10 & $61.80 \pm 8.05$ & $9(90 \%)$ & $7(70 \%)$ & $24.31 \pm 3.03$ \\
III & 15 & $63.33 \pm 8.26$ & $15(100 \%)$ & $6(40 \%)$ & 2.93 \\
IV & 8 & $62.50 \pm 9.98$ & $7(87.5 \%)$ & $5(62.5 \%)$ & $3.13 \pm 1.36$ \\
\hline
\end{tabular}

SUI, stress urinary incontinence; BMI, body mass index.

paraffin-embedded and dehydrated by standard methods. The sample was then sectioned into $3-\mu \mathrm{m}$ sections in preparation for immunohistochemistry.

Immunohistochemistry. Each section was smoothed and placed onto a polylysine-coated slide. Slides were incubated overnight in a $57^{\circ} \mathrm{C}$ oven, deparaffinized by xylene washes ( $3 \times 5 \mathrm{~min})$, and hydrated with serial ethanol solutions ( $3 \times 3$ min). The slides were then placed in $d_{d H_{2}} \mathrm{O}$ prior to being transferred into citrate buffer $(\mathrm{pH}$ 6.0) and heated to $92-97^{\circ} \mathrm{C}$ for $15 \mathrm{~min}$. Slides were subsequently removed and cooled for $5 \mathrm{~min}$ at room temperature. Each slide was covered with a drop of $3 \%$ peroxide, incubated at room temperature for 15 min to block endogenous peroxidase, and then washed with phosphate-buffered saline (PBS) 3x5 min. Slides were incubated overnight at $4^{\circ} \mathrm{C}$ with $50 \mu \mathrm{l}$ of a solution containing rabbit anti-human NPY polyclonal antibody (Millipore, Billerica, MA, USA) and then washed with PBS 3x5 min. Slides were then incubated with a secondary antibody ( $\operatorname{IgG})$ at room temperature for $2 \mathrm{~h}$ and washed with PBS 3x5 min. Afterwards, the slides were treated with a horseradish peroxidase-labeled tertiary antibody, incubated at room temperature for $45 \mathrm{~min}$, and washed with PBS $3 \times 5 \mathrm{~min}$. Each slide was then covered with $100 \mu \mathrm{l}$ freshly prepared DAB color liquid, incubated at room temperature for 5-10 min, and examined for color development under a microscope. Finally, slides were washed with tap water, re-stained with hematoxylin, dehydrated in an ethanol series, treated with xylene for clearing and mounted in permount. Each batch of color liquid was monitored with positive and negative controls, human brain tissue and PBS, respectively.

Semi-quantitative evaluation of the detection results. The distribution of NPY in the mucosa of the anterior vaginal wall was evaluated by immunohistochemistry. Under a high magnification microscope, samples were scored as follows: negative $(-)$, no brown coloration; weak positive $(+)$, sparse and light brown coloration; positive $(++)$, dense brown coloration; strong positive $(+++)$, intense brown coloration.

Statistical analysis. Experimental results were analyzed using SPSS 13.0 software. All data (age, parity and BMI) were presented as the means \pm SD. Spearman rank correlation analysis was performed to elucidate the correlation between NPY expression variation and POP stage. Multivariate regression analysis was used to examine the correlation between the
Table II. Comparison of NPY expression in the anterior vaginal wall tissue of patients (based on immunohistochemistry).

\begin{tabular}{lrrrrr}
\hline \multirow{2}{*}{ Group } & \multirow{2}{*}{ Cases } & \multicolumn{4}{c}{ Staining inensity } \\
\cline { 3 - 6 } & & - & + & ++ & +++ \\
\hline Control & 9 & 0 & 0 & 2 & 7 \\
POP I & 8 & 0 & 0 & 5 & 3 \\
POP II & 10 & 3 & 6 & 1 & 0 \\
POP III & 15 & 8 & 6 & 1 & 0 \\
POP IV & 8 & 5 & 3 & 0 & 0 \\
POP II + SUI & 6 & 2 & 4 & 0 & 0 \\
POP III + SUI & 6 & 5 & 1 & 0 & 0 \\
POP IV + SUI & 5 & 5 & 0 & 0 & 0 \\
\hline
\end{tabular}

POP, pelvic organ prolapse; SUI, stress urinary incontinence.

pathogenic factors of SUI complication (age, parity and BMI) and NPY expression.

\section{Results}

Distribution pattern of NPY. The control group showed clear NPY expression, not only in the adventitia of blood vessels but also in the surrounding glands and smooth muscle. Notably, certain foci with strong staining were composed of networks of NPY-positive nerve fibers and circles of adventitia covering blood vessels. In areas of strong NPY expression in POP I samples, NPY was observed in dotted lines decorating the adventitia. As the severity of disease intensified, we observed fewer and weaker circles of NPY staining. We also observed less NPY expression in the connective tissue of patients with higher staged disease. Samples from the POP IV or POP/SUI groups showed only weak, sparse NPY staining. In samples from the POP IV, POP III/SUI and POP IV/SUI groups, the anterior vaginal wall displayed the lowest level of NPY expression, which was barely detectable (Fig. 1).

Semi-quantitative evaluation of NPY. Semi-quantitative evaluation of NPY positive staining in anterior vaginal wall tissue in POP and control groups is shown in Table II. 

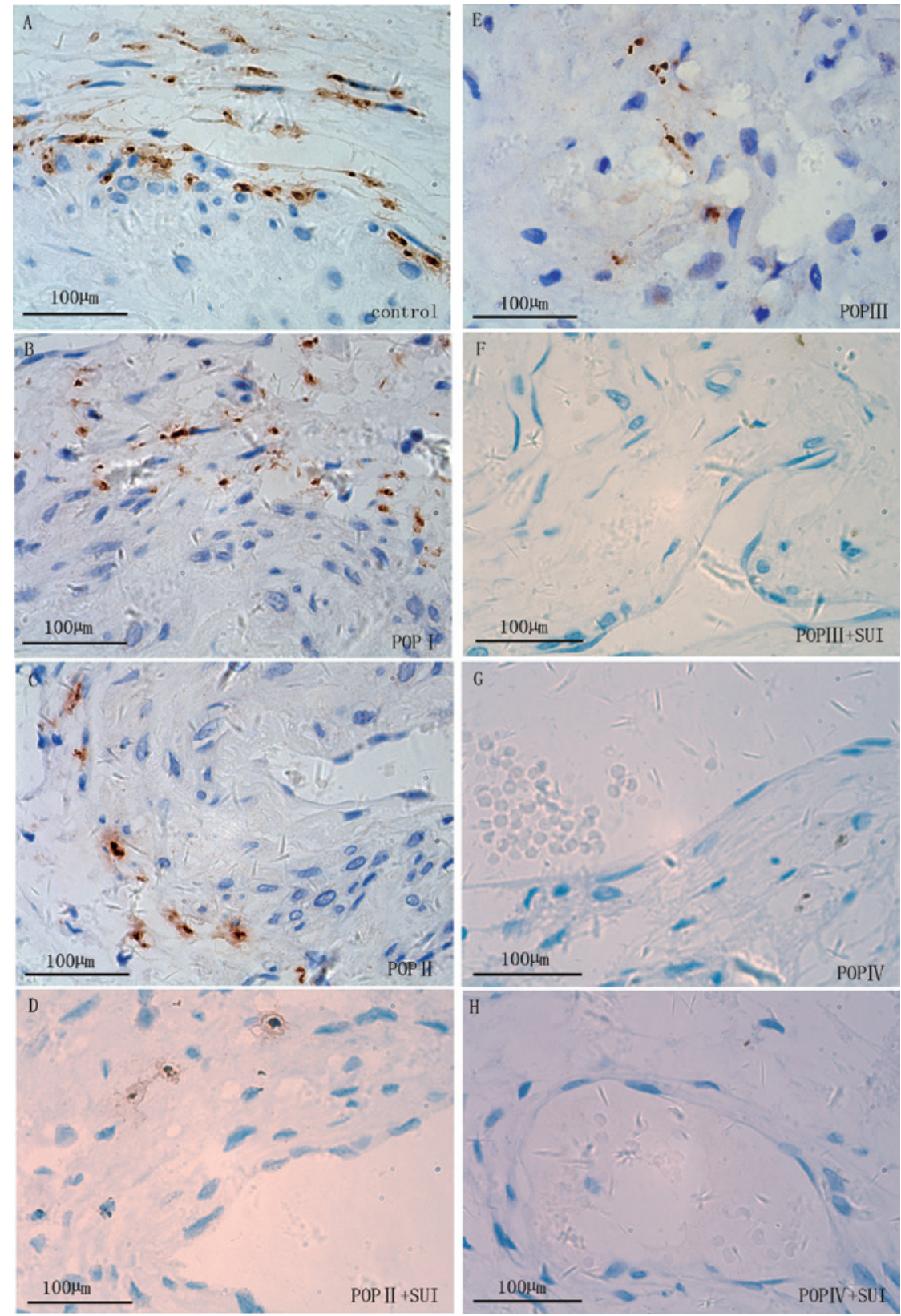

Figure 1. Neuropeptide Y (NPY) immunohistochemistry in the anterior vaginal wall tissue. (A) Control group. Intensive network of nerve fibers decorated with brown staining, indicating high NPY expression appearing on adventitia, surrounding glands and smooth muscle. Adventitia covering blood vessels manifested as circles of brown staining (x1000). (B) POP I group. Dotted brown NPY staining was visible (x1000). (C) POP II group. Sparse brown NPY staining was found on blood vessels and surrounding glands (x1000). (D) POP II/SUI group. Sparse dots of NPY brown staining were found on blood vessels and surrounding glands (x1000). (E) POP III group. Few instances of brown NPY staining could be found on blood vessels and surrounding glands (x1000). (F) POP III/SUI group. Few instances of brown NPY staining were present on blood vessels and surrounding glands (x1000). (G) POP IV group. Light yellow staining of NPY was not detected (x1000). (H) POP IV/SUI group. Light yellow NPY staining was not detected (x1000).

Analysis of NPY-POP correlation. Analysis of Spearman rank correlation revealed a significant negative correlation between NPY expression level and POP severity $(r=-0.778$, $\mathrm{P}<0.001$ ).

Assessment of the correlation between NPY and POP pathogenic factors. Since patients in all groups showed a similar age distribution, age was not analyzed as a contributing factor (Table III). These results show that menopause and SUI complications display significant correlations with NPY expression level $(\mathrm{P}<0.05)$, whereas parity and BMI appear to be unrelated.

\section{Discussion}

NPY-POP relationship. NPY, a 36-amino-acid neuropeptide, is the most abundant neuropeptide in the vagina (2) with a NPY-positive neuropil underlying the vaginal epithelium. NPY-positive nerve fibers are also distributed throughout the vaginal blood vessels and non-vascular smooth muscle (3). NPY is also widespread in the perivascular area and is associated with tension in the small vascular wall, contributing to the contraction of the small arteries. As such, NPY is capable of mediating the neural regulation of local blood flow in the reproductive tract. Using semi-quantitative immu- 
Table III. Multivariate regression analysis of NPY and POP pathogenic factors.

\begin{tabular}{|c|c|c|c|c|c|c|c|c|}
\hline & \multirow[t]{2}{*}{ Group } & \multirow[t]{2}{*}{ Estimate } & \multirow{2}{*}{$\begin{array}{l}\text { Standard } \\
\text { error }\end{array}$} & \multirow[t]{2}{*}{ Wald } & \multirow[t]{2}{*}{$\mathrm{v}$} & \multirow[t]{2}{*}{ P-value } & \multicolumn{2}{|c|}{$95 \% \mathrm{CI}$} \\
\hline & & & & & & & $\begin{array}{l}\text { Lower } \\
\text { bound }\end{array}$ & $\begin{array}{l}\text { Upper } \\
\text { bound }\end{array}$ \\
\hline \multirow[t]{3}{*}{ Threshold } & $\begin{array}{l}\text { (Immunohistochemistry-based } \\
\text { NPY expression, 0) }\end{array}$ & -5.574 & 1.134 & 24.149 & 1 & $<0.001$ & -7.797 & -3.351 \\
\hline & $\begin{array}{l}\text { (Immunohistochemistry-based } \\
\text { NPY expression, 1) }\end{array}$ & -2.874 & 0.901 & 10.162 & 1 & 0.001 & -4.640 & -1.107 \\
\hline & $\begin{array}{l}\text { (Immunohistochemistry-based } \\
\text { NPY expression, 2) }\end{array}$ & -0.764 & 0.726 & 1.107 & 1 & 0.293 & -2.187 & 0.659 \\
\hline \multirow[t]{7}{*}{ Location } & Parity & -0.157 & 0.321 & 0.239 & 1 & 0.625 & -0.786 & 0.472 \\
\hline & Menopause-positive & -3.005 & 0.911 & 10.887 & 1 & 0.001 & -4.791 & -1.220 \\
\hline & Menopause-negative & 0 & - & - & 0 & - & - & - \\
\hline & SUI complication & -3.634 & 0.867 & 17.562 & 1 & $<0.001$ & -5.333 & -1.934 \\
\hline & No SUI complication & 0 & - & - & 0 & - & - & - \\
\hline & $\mathrm{BMI}>23$ & 0.026 & 0.679 & 0.002 & 1 & 0.969 & -1.305 & 1.358 \\
\hline & BMI $<23$ & 0 & - & - & 0 & - & - & - \\
\hline
\end{tabular}

NPY, neuropeptide Y; SUI, stress urinary incontinence; BMI, body mass index.

nohistochemistry to examine biopsy specimens of the human pubococcygeus muscle, Busacchi et al showed that neuropeptide staining is reduced in POP patients and, furthermore, that it was most reduced in POP III/SUI patients, correlating loss of NPY with disease severity (4). Subsequent studies using RT-PCR examined the mRNA level of NPY-Y1 and NPY-Y2 receptors in cardinal and uterosacral ligaments from $\mathrm{POP}$ patients and found that NPY is systematically involved in POP pathogenesis (5). In 2009, Zhang et al showed that NPY is reduced in the uterosacral ligament system and anterior vaginal wall in cases where the pelvic floor is relaxed (6). This suggests that nerve injury to the pelvic floor plays a crucial role in POP development. In this study, we showed that NPY expression is negatively correlated with POP severity, suggesting that NPY may play a role in the localized neuromodulation of blood flow in the reproductive tract. Loss of NPY therefore may result in reduced angiogenesis and insufficient blood supply, leading to weak muscles and connective tissue in the pelvic floor. It is possible that the level of nerve damage in POP patients is associated with reduced NPY expression in the anterior vaginal wall. An assessment of NPY expression may be employed to predict the likelihood of developing POP.

Effect of POP pathogenic factors on NPY innervation. Using multivariate regression analysis, we found that menopause and SUI complications have greater impacts on NPY reduction than other indices, such as parity, age and BMI. It is possible that menopause and SUI inflict significant injuries on NPY-positive nerve fibers. Following menopause, estrogen levels drop to a basal level, reducing growth stimulation of the urogenital tract epithelium and suppressing blood supply in the submucosal plexus (7-9). These factors interfere with the metabolism and function of connective tissue in the reproductive tract. It is possible that the marked reduction in estrogen following menopause also inhibits innervation and decreases NPY distribution. NPY plays a pivotal role in the occurrence and development of POP. Studies have shown changes in neuropeptide content in certain pelvic floor tissues (levator ani and the vaginal wall) in patients with POP and SUI. Hong et al examined the vaginal neuropeptide expression profile in 61 perimenopausal POP and SUI patients and discovered that the expression and content of VIP is significantly reduced and that the reduction is negatively correlated with age, menopause and parity (1). A number of electrophysiological studies, based on nerve electrophysiology and electromyography, have confirmed that childbirth, menopause and SUI cause nerve damage to the pelvic floor (10-12).

POP occurrence, nerve damage and neurotransmitter reduction are generally thought to be responsible for the vascular changes in and hypoperfusion of the pelvic floor. These factors cause changes in the metabolism of supportive structures and an alteration of constituents (13). One might think of the anterior vaginal wall as a component of the pelvic 'hammock'. A decrease in neurotransmitter in the anterior vaginal wall may lead to a change in 'hammock' tension, thereby causing SUI. POP is closely associated with SUI; $80 \%$ of SUI patients suffer POP complications, and $50 \%$ of POP patients have concurrent SUI (14). In the current study, we observed no SUI complications in the least severe group (POP I). However, $70 \%$ of patients in POP II (7/10), 40\% in POP III (6/15), and 62.5\% in POP IV $(5 / 8)$ had concurrent SUI complications. The average of SUI complications across the POP II, III and IV groups was 55\% $(18 / 33)$. It is not clear whether the ratio of SUI complications in POP patients is related to severity of the POP disease. Further studies with larger sample sizes are required to clarify this issue. Notably, POP II/SUI and III/SUI patients exhibit lower NPY expression in the anterior vaginal wall than their POP II and III counterparts. Since POP III and IV patients have late stage 
pelvic floor dysfunction, it is likely that the denervated injuries in these patients are too severe to be repaired. Additionally, SUI and urine voiding dysfunction may be associated with prolapse of the anterior wall and top of the vagina. Thus, POP III and IV patients are more vulnerable to concurrent SUI (15). POP and SUI also have overlapping symptoms in denervated injuries, which may mask the early symptoms of SUI. For example, as POP progressively intensifies, prolapse of the anterior vaginal wall distorts the urethra, resulting in mechanical obstruction and decreased urine leakage (16). Nevertheless, the denervated injuries of POP and SUI are additive and lead to a tremendous reduction in NPY expression. This suggests that the clinical symptoms exhibited by patients do not always match those of the disease development. However, when pelvic floor dysfunction diseases reach an advanced stage, they all manifest as the urinary symptoms of frequent micturition, urgency micturition and SUI.

In this study, we observed that neuropeptide loss and reduction in the reproductive tract of POP/SUI patients are both involved in pathogenesis. Neuropeptides play a role in modulating capillary permeability, thereby affecting blood and nutrient supply and metabolic changes in local tissues (17). In effect, loss of neuropeptides causes local malnutrition and atrophy of muscle and connective tissue. We speculate that once POP progresses to a certain level, nerve injuries are irreversible. We also believe SUI occurrence further aggravates POP damage. Further studies are required to address whether POP complications, in addition to SUI, may result in similar consequences.

It is notable that SUI appears first in certain patients and POP emerges first in others, despite their similar pathogenic basis. The underlying physiological and pathological mechanisms of these diseases remain unclear but are likely related to nerve damage. This study revealed a connection between POP diseases and NPY reduction in peptidergic nerve fibers. Further studies are necessary to determine the differences and similarities between SUI and POP, thereby providing a basis for clinical treatment.

\section{References}

1. Hong X, Huang L and Song Y: Role of vasoactive intestinal peptide and pituitary adenylate cyclase activating polypeptide in the vaginal wall of women with stress urinary incontinence and pelvic organ prolapse. Int Urogynecol J Pelvic Floor Dysfunct 19: 1151-1157, 2008.
2. Ottesen B: Vasoactive intestinal polypeptide as a neurotransmitter in the female genital tract. Am J Obstet Gynecol 147: 208-224, 1983.

3. Sievert KD, Bakircioglu ME, Tsai T, Nunes L and Lue TF: The effect of labor and/or ovariectomy on rodent continence mechanism - the neuronal changes. World J Urol 22: 244-250, 2004.

4. Busacchi P, De Giorgio R, Santini D, et al: A histological and immunohistochemical study of neuropeptide containing somatic nerves in the levator ani muscle of women with genitourinary prolapse. Acta Obstet Gynecol Scand 78: 2-5, 1999.

5. Jiang Fang LJ and Chen J: Neurotransmitters in pelvic floor support tissues in pelvic organ prolapse patients. Peking Union Medical College, 2006.

6. Zhang L and Ma LX: Neuropeptide Y expression in pelvic tissues and blood plasma and its relation to pelvic floor relaxation. Jinan University, 2009.

7. Goodman Y, Bruce AJ, Cheng B and Mattson MP: Estrogens attenuate and corticosterone exacerbates excitotoxicity, oxidative injury, and amyloid beta-peptide toxicity in hippocampal neurons. J Neurochem 66: 1836-1844, 1996.

8. Chaban VV, Mayer EA, Ennes HS and Micevych PE: Estradiol inhibits atp-induced intracellular calcium concentration increase in dorsal root ganglia neurons. Neuroscience 118: 941-948, 2003.

9. Kane DD, Kerns JM, Lin DL and Damaser MS: Early structural effects of oestrogen on pudendal nerve regeneration in the rat. BJU Int 93: 870-878, 2004

10. Dietz HP and Wilson PD: Childbirth and pelvic floor trauma. Best Pract Res Clin Obstet Gynaecol 19: 913-924, 2005.

11. Pierce LM, Baumann S, Rankin MR, et al: Levator ani muscle and connective tissue changes associated with pelvic organ prolapse, parity, and aging in the squirrel monkey: a histologic study. Am J Obstet Gynecol 197: 60.e61-e69, 2007.

12. Jozwik M and Jozwik M: Partial denervation of the pelvic floor during term vaginal delivery. Int Urogynecol J Pelvic Floor Dysfunct 12: 81-82, 2001

13. Hu J and Zhou LH: The relationship of pelvic organ prolapse and neuropeptides (VIP, NPY). Progress of Anatomical Sciences: 467-470, 2010.

14. Bai SW, Jeon MJ, Kim JY, Chung KA, Kim SK and Park KH: Relationship between stress urinary incontinence and pelvic organ prolapse. Int Urogynecol J Pelvic Floor Dysfunct 13: 256-260, 2002

15. Busacchi P, Perri T, Paradisi R, et al: Abnormalities of somatic peptide-containing nerves supplying the pelvic floor of women with genitourinary prolapse and stress urinary incontinence. Urology 63: 591-595, 2004

16. Dietz HP, Clarke B and Vancaillie TG: Vaginal childbirth and bladder neck mobility. Aust NZJ Obstet Gynaecol 42: 522-525, 2002.

17. Kerns JM, Damaser MS, Kane JM, et al: Effects of pudendal nerve injury in the female rat. Neurourol Urodyn 19: 53-69, 2000. 